\title{
DIVERSITY AND ACTIVITY OF INSECT NATURAL ENEMIES OF THE BAGWORM (Lepidoptera: Psychidae) WITHIN AN OIL PALM PLANTATION IN PERAK, MALAYSIA
}

\author{
NORMAN KAMARUDIN* and OTHMAN ARSHAD*
}

\begin{abstract}
Bagworms (Lepidoptera: Psychidae) are one of the important leaf-eating pests of oil palm in Malaysia and Indonesia. Crop losses, due to the extensive defoliation by a serious bagworm attack are inevitable. The establishment and propagation of the bagworm's parasitoids, depend very much on species of flowering plants as sources of nectar. Cassia cobanensis, was proven suitable due to its attractiveness to most bagworm parasitoids. A study to assess the diversity of bagworm's natural enemies (hymenopterous parasitoids and reduuvid predatory bugs) on the C. cobanensis plant and within the undergrowth of the oil palm planting block was evaluated by sweep net and sticky trap sampling. Environmental parameters such as light intensity, temperature and relative humidity were also recorded during the sampling to determine their possible range of influence towards the activity of the insect natural enemies. The ShannonWiener diversity index of insect natural enemies occurring on the C. cobanensis plant was 2.32. Among the dominant parasitoids observed on $\mathrm{C}$. cobanensis plants are chalcids (Brachymeria lugubris and Brachymeria carinata) and braconids (Dolichogenidea metesae and Apanteles aluella), besides the reduuvid predator, Cosmolestes picticeps. In contrast, within the oil palm planting block, the undergrowth within the non-harvesting path only recorded a much lower diversity index of 1.09 and 1.12 each, in a block with C. cobanensis at the roadside and without, respectively. However, C. picticeps was shown to be much more dominant within both oil palm planting blocks, up to two- to three-fold, with much lesser numbers of hymenopterous parasitoids (less than five individuals). This indicates the important contribution of $\mathrm{C}$. cobanensis plant towards enriching the diversity of the parasitoids, which are normally not found within the oil palm block, due to much less intense sunlight. In terms of insect activity, they are significantly more active in the moderate or medium light intensities $(<8000 \mathrm{fc})$, medium humidity levels (50\%-69\%) and medium temperatures $\left(30^{\circ} \mathrm{C}-34^{\circ} \mathrm{C}\right)$.
\end{abstract}

Keywords: bagworm, Pteroma pendula, natural enemies, diversity, environment, oil palm.

Date received: 22 April 2016; Sent for revision: 2 June 2016; Received in final form: 9 August 2016; Accepted: 10 August 2016.

Malaysian Palm Oil Board

6 Persiaran Institusi, Bandar Baru Bangi, 43000 Kajang,

Selangor, Malaysia.

E-mail: norman@mpob.gov.my

\section{INTRODUCTION}

Bagworms (Lepidoptera: Psychidae) are one of the important leaf-eating pests of oil palm in Malaysia and Indonesia. Crop losses, due to the 
extensive defoliation by a serious bagworm attack are inevitable. A moderate defoliation of about $10 \%-13 \%$ may cause a crop loss of about $33 \%-40 \%$ (Basri, 1993). Parasitoids play an important role in regulating bagworm numbers. The propagation and establishment of the insect natural enemies depend on some suitable species of flowering plants as sources of nectar. Basri et al. (1999) confirmed that flowering plants prolong the life span of the adult parasitoids in the laboratory. Among all beneficial plants, Cassia cobanensis (Fabaceae) is deemed most suitable for propagation in oil palm estates due to its easy establishment and maintenance. Ho (2003) has confirmed that C. cobanensis and Euphorbia heterophylla (Euphorbiaceae) are comparable in terms of being attractive to parasitoids in the field. However C. cobanensis has the competitive edge, of which it is easier to propagate and need not be successively replanted at every three months as with E. heterophylla. A more recent study (Nor Sarashimatun et al., 2011), has shown that C. cobanensis is second best to E. heterophylla in terms of attractiveness to bagworm parasitoids, followed by Turnera subulata (Passifloraceae), Antigonon leptopus (Polygonaceae), Vitis japonica (Vitaceae) and Turnera ulmifolia (Passifloraceae).

An inventory of insect natural enemies affecting the bagworms and nettle caterpillars in oil palm has been documented previously (Norman et al., 1996; 1998). This current study intends to assess the diversity of insect natural enemies of the bagworm, found occurring on the beneficial plant (C. cobanensis) and within the oil palm planting block. At the same time, the range of environmental parameters which influence the activities of these natural enemies within the oil palm ecosystem are also studied.

\section{MATERIALS AND METHODS}

An oil palm plantation infested with the bagworm, Pteroma pendula was selected at Telok Intan, Perak, Malaysia. Two oil palm planting blocks were selected: one having three groves of the beneficial plant, C. cobanensis planted at the roadside, while another, without $C$. cobanensis planted at its vicinity, was used as a control. Each block was about 5 ha, with palm age of 3-4 years old at the start of the study.

Sweep net samplings were conducted on the C. cobanensis groves, by sweeping four times above the grove quickly and turning the opening from side to side, to capture the insects within the beneficial plant. This method of sampling is replicated three times in a completely randomised design (CRD). Samples were taken at around $9.00 \mathrm{am}$ and 12 noon, for two consecutive days, in each sampling period. Environmental parameters such as light intensity (foot-candles, fc), temperature $\left({ }^{\circ} \mathrm{C}\right)$ and relative humidity $(\% \mathrm{RH})$, were recorded during the sampling period.

A square sticky trap, made from plywood and painted yellow, was covered with a plastic sleeve and sprayed with polybutene gum (Brand: NEOPEACE-F101 - Polybutene - $16 \% \mathrm{w} / \mathrm{w}$ ) on both surfaces to capture free flying insects above the $C$. cobanensis grove. The sticky trap was placed at about $1.5 \mathrm{~m}$ above the ground, in the middle of the beneficial plant grove and left in the field for two days. The traps were replicated three times in a CRD among the $C$. cobanensis groves. The plastic sleeve was collected and replaced the following morning (9.00 am). This was done for two consecutive days.

Within each of the two planting blocks, sweep net sampling was also conducted at the non-harvesting path (sweeping four times while walking along the path) and replicated three times at random within the oil palm planting block. At the same time, three sticky traps were also placed randomly at the path. Samples were gathered similarly as conducted on the C. cobanensis plant. Data was collected quarterly from 2008 to 2010.

In these sampling, it was deduced that the sweep net captures resemble the resident population within the non-harvesting path or within the C. cobanensis plant. Whereas, sticky trap captures should resemble the activity of the insects as they were caught while hovering and flying around the beneficial plant or among the non-harvesting paths within the oil palm planting block.

All samples were brought to the laboratory for sorting and identification of common bagworm parasitoids and predators to bagworms and nettle caterpillars following Norman et al. (1996; 1998). Shannon-Wiener biodiversity indices were calculated using the online programme at https: / / www.easycalculation.com / statistics / shannonwiener-diversity.php

\section{RESULTS}

\section{Diversity of Insect Natural Enemies in the Oil Palm Ecosystem}

Cassia cobanensis plant. Figure 1 shows the overall diversity of insect natural enemies occurring on the Cassia cobanensis plant, collected by both sweep net and sticky traps. The Shannon-Wiener diversity index was 2.32. For the hymenopterous parasitoids, there were three species each from the families Ichneumonidae (Spinaria spinator and Paraphylax varius), Eulophidae (Tetrastichus sp., Sympiesis sp. and Pediobius imbreus) and Chalcididae (Brachymeria lasus, B. carinata and B. lugubris); two species from the family Braconidae 
(Dolichogenidea metesae and Apanteles aluella) and one species each from the families Eurytomidae (Eurytoma sp.), Eupelmidae (Eupelmus catoxanthae), and Ceraphronidae (Aphanogmus thylax). Apart from parasitoids, the two common species of predators (Hemiptera: Reduviidae) captured were Sycanus dichotomus and Cosmolestes picticeps.

It was observed that within the $C$. cobanensis plant, chalcids (B. lugubris and B. carinata) appeared to be much more dominant, followed by the braconids (A. aluella and D. metesae) (Figure 1). There were three species of parasitoids (E. catoxanthae,
P. imbreus and B. lasus found on the C. cobanensis plant (Figure 1) but not detected in the oil palm planting blocks (Figures 2 and 3). S. dichotomus was also found only on the C. cobanensis plant (Figure 1).

Within oil palm block with Cassia cobanensis at the roadside. It was noted that within the oil palm planting block, even with having Cassia groves at the roadside, the Shannon-Wiener index of diversity has reduced to 1.10 , compared to 2.32 on the $C$. cobanensis plant. Figure 2 shows that there were two species of hymenopterous parasitoids from the family

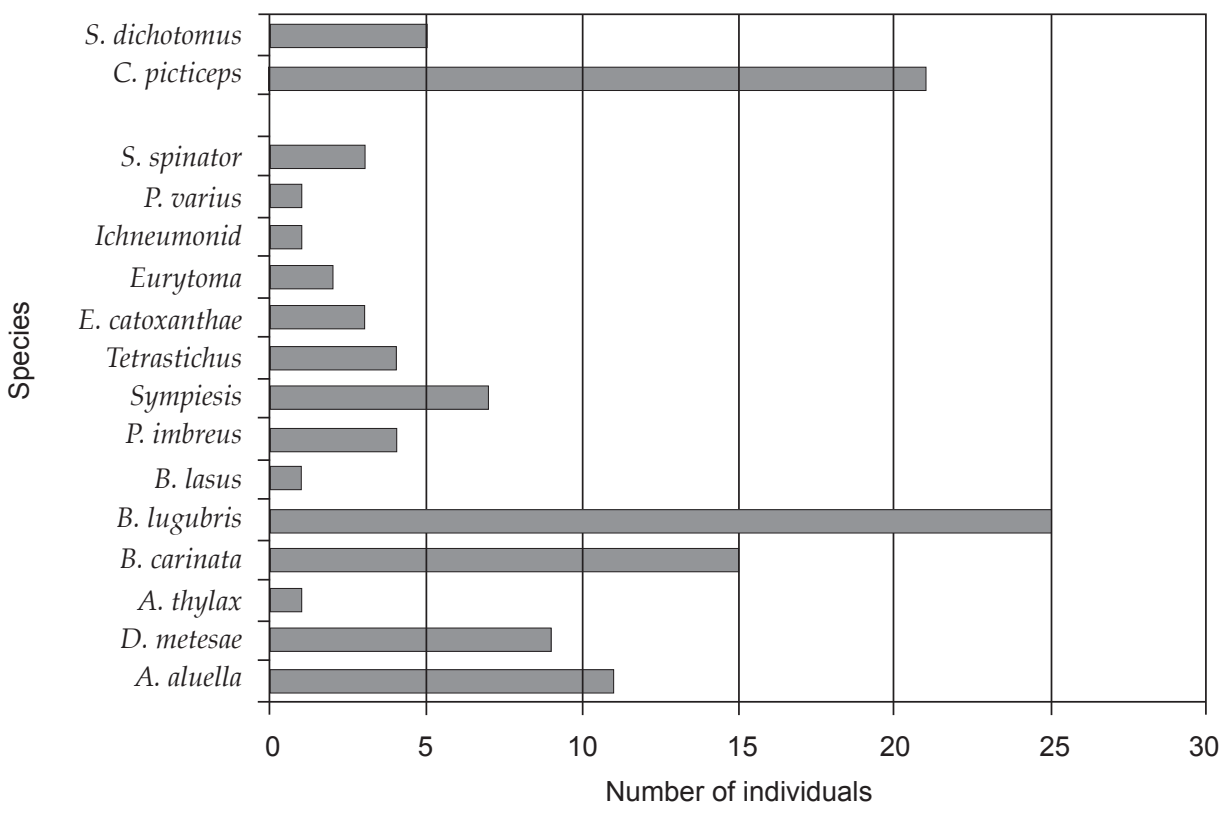

Species richness: 16 , Shannon diversity index: 2.32

Figure 1. Biodiversity of bagworm natural enemies on Cassia cobanensis plant, at a plantation in Telok Intan, Perak, Malaysia.

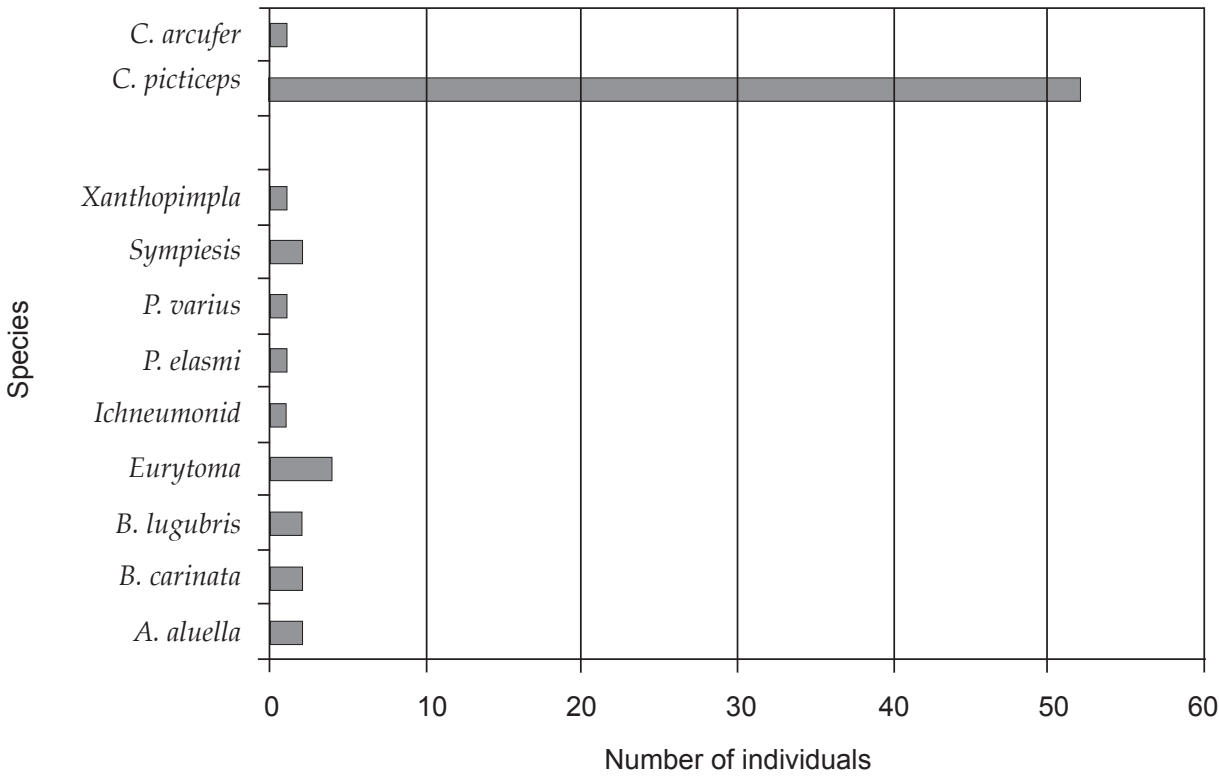

Species richness: 11 , Shannon diversity index: 1.10

Figure 2. Biodiversity of bagworm natural enemies within the oil palm block (with C. cobanensis at the roadside) at Telok Intan, Perak, Malaysia. 
Ichneumonidae (Xanthopimpla sp. and Paraphylax varius), two species each from Eulophidae (P. elasmi and Sympiesis sp.) and Chalcididae (B. lugubris and B. carinata) and one species each from Eurytomidae (Eurytoma sp.) and Braconidae (A. aluella). The dominant insect within the area was the reduuvid predator, C. picticeps. This species was captured more than one-fold than those on $C$. cobanensis (Figures 1 and 2). A clerid predator, Callimerus arcufer was also captured. Eurytoma sp. was captured more frequently than others, with number of parasitoids not exceeding five individuals for each species (Figure 2). Xanthopimpla sp. P. elasmi and C. arcufer were found in this block which were not detected on the C. cobanensis plant (Figures 1 and 2).

Within oil palm block without Cassia cobanensis at the roadside (control). The captures of insect parasitoids in the oil palm planting block without any nearby presence of $C$. cobanensis plants was also low (less than five individuals per species). Only three species of parasitoids appeared to be captured more frequently than others (B. lugubris, D. metesae and $S$. spinator). However, the reduviid predator was also dominant, with more than two-fold the number captured on C. cobanensis plant (Figure 3). The Shannon-Wiener diversity index (1.12) is almost similar to the planting block having the Cassia plant at the roadside (1.10). Similarly, it was also relatively much lower than recorded on the $C$. cobanensis plant (2.32). In terms of parasitoid species composition, there were three species of Eulophidae (Tetrastichus sp., Sympiesis sp. and P. anomalus), two species each of Chalcididae (B. lugubris and
B. carinata), Braconidae (D. metesae and A. aluella), Ichneumonidae ( $S$. spinator and $P$. varius) and one species of Ceraphronidae (A. thylax).

\section{Effects of Environmental Parameters on Activity of Natural Enemies}

Insect activities in relation to light intensity $\left(f_{c}\right)$. With sticky traps, the activities of most insect natural enemies were observed highest between light intensities of 2000-4999 fc (Table 1). Activities by insects were significantly lower at high light intensities (>8000 fc) (Table 3).

The braconid, $A$. aluella seem to be more active at lower light intensities of between 2000-3000 fc (Table 1). A significant correlation $\left(\mathrm{r}^{2}=0.84, \mathrm{p}<0.05\right)$ was detected for the captures of A. aluella using sticky traps (indicating activity) with light intensity. Similarly, although ichneumonids can be active over a broad range of light intensities (1000-13 000 fc), $P$. varius seems to be more active in lower light intensity between 2000-3000 fc. S. spinator seems to be active in less intense sunlight, between 2000$4000 \mathrm{fc}$ (Table 1). D. metesae on the other hand, appeared to have periodic activities between 300$1000 \mathrm{fc}, 3000-4000 \mathrm{fc}$ and 6000-7000 fc. Chalcids (B. lugubris) appeared active between 2000-3000 fc while $B$. carinata seem to be active at 1000-2000 fc and 3000-4000 fc (Table 1). Eulophids (P. anomalus, P. imbreus, Sympiesis and Tetrastichus) were observed more active at higher light intensities of between 4000-5000 fc (Table 1).

By using sweep net, most of the natural enemies were caught at the lower range of light intensity

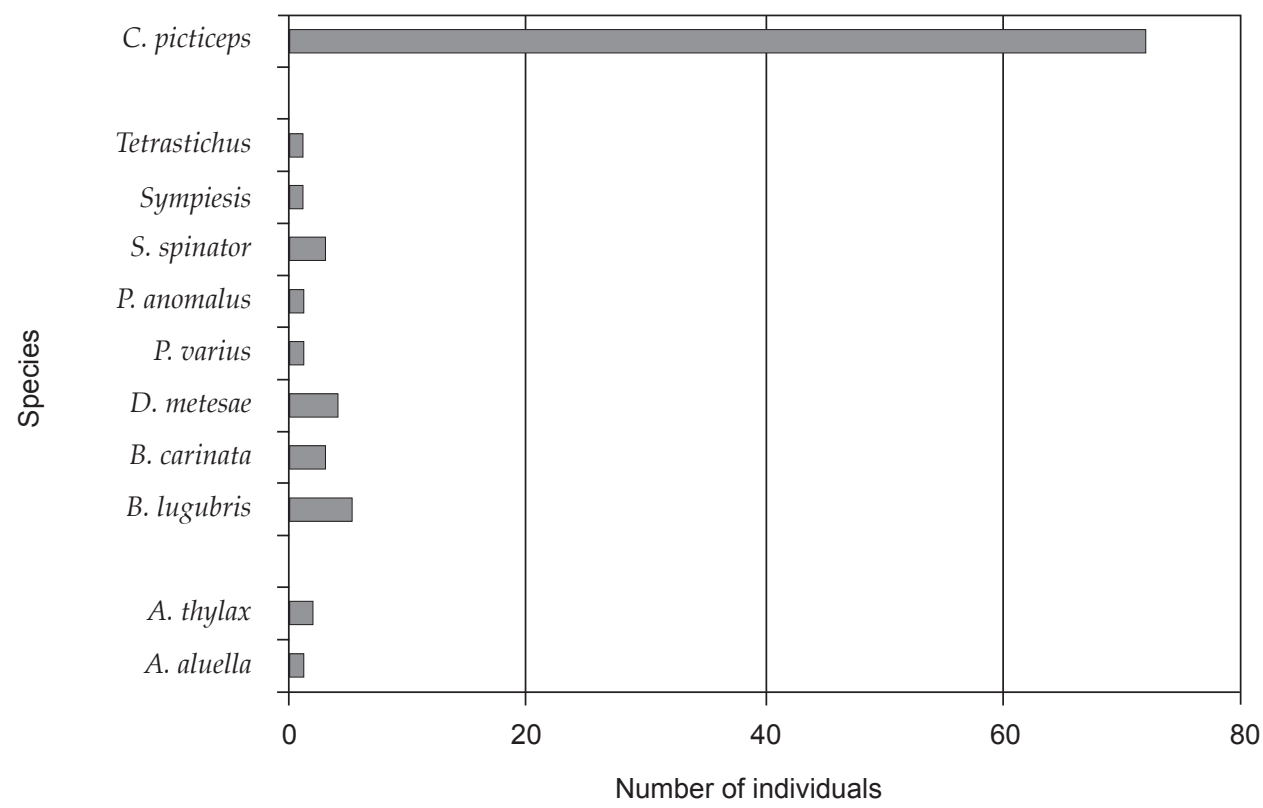

Species richness: 12, Shannon diversity index: 1.12

Figure 3. Biodiversity of bagworm natural enemies within the oil palm block (without C. cobanensis at the roadside) at Telok Intan, Perak, Malaysia. 
TABLE 1. DISTRIBUTION OF NATURAL ENEMIES' ACTIVITY (sticky trap) ALONG VARIOUS LIGHT INTENSITIES

\begin{tabular}{|c|c|c|c|c|c|c|c|c|c|c|c|c|}
\hline \multirow[b]{3}{*}{ Species } & \multirow[b]{3}{*}{ Order } & \multicolumn{11}{|c|}{ No. of individuals captured by sticky traps } \\
\hline & & \multicolumn{11}{|c|}{ Light intensity (fc) } \\
\hline & & $\begin{array}{c}300- \\
999\end{array}$ & $\begin{array}{c}1000- \\
1999\end{array}$ & $\begin{array}{c}2000- \\
2999\end{array}$ & $\begin{array}{l}3000- \\
3999\end{array}$ & $\begin{array}{c}4000- \\
4999\end{array}$ & $\begin{array}{c}5000- \\
5999\end{array}$ & $\begin{array}{c}6000- \\
6999\end{array}$ & $\begin{array}{c}7000- \\
7999\end{array}$ & $\begin{array}{c}8000- \\
8999\end{array}$ & $\begin{array}{l}9000- \\
9999\end{array}$ & $\begin{array}{c}10000- \\
13000\end{array}$ \\
\hline A. aluella & Braconidae & 0.00 & 1.00 & 7.00 & 0.00 & 6.00 & 4.00 & 5.00 & 1.00 & 1.00 & 0.00 & 0.00 \\
\hline D. metesae & Braconidae & 2.00 & 0.00 & 1.00 & 2.00 & 1.00 & 1.00 & 2.00 & 0.00 & 0.00 & 0.00 & 0.00 \\
\hline S. spinator & Braconidae & 0.00 & 0.00 & 2.00 & 2.00 & 1.00 & 0.00 & 0.00 & 1.00 & 0.00 & 0.00 & 0.00 \\
\hline A. thylax & Ceraphronidae & 0.00 & 0.00 & 0.00 & 1.00 & 2.00 & 0.00 & 1.00 & 0.00 & 1.00 & 0.00 & 0.00 \\
\hline B. lugubris & Chalcididae & 0.00 & 0.00 & 2.00 & 0.00 & 0.00 & 0.00 & 0.00 & 0.00 & 0.00 & 0.00 & 0.00 \\
\hline B. carinata & Chalcididae & 0.00 & 1.00 & 0.00 & 1.00 & 0.00 & 1.00 & 0.00 & 0.00 & 0.00 & 0.00 & 0.00 \\
\hline C. arcufer & Cleridae & 0.00 & 0.00 & 1.00 & 1.00 & 0.00 & 1.00 & 1.00 & 0.00 & 0.00 & 0.00 & 0.00 \\
\hline P. imbreus & Eulophidae & 0.00 & 1.00 & 2.00 & 2.00 & 3.00 & 0.00 & 0.00 & 0.00 & 0.00 & 0.00 & 0.00 \\
\hline P. anomalus & Eulophidae & 0.00 & 0.00 & 1.00 & 1.00 & 2.00 & 0.00 & 0.00 & 0.00 & 0.00 & 0.00 & 0.00 \\
\hline Sympiesis & Eulophidae & 0.00 & 0.00 & 1.00 & 0.00 & 2.00 & 0.00 & 1.00 & 0.00 & 0.00 & 0.00 & 0.00 \\
\hline Tetrastichus & Eulophidae & 0.00 & 0.00 & 1.00 & 0.00 & 1.00 & 0.00 & 0.00 & 0.00 & 0.00 & 1.00 & 0.00 \\
\hline E. catoxanthae & Eupelmidae & 0.00 & 1.00 & 0.00 & 0.00 & 1.00 & 1.00 & 0.00 & 0.00 & 0.00 & 0.00 & 0.00 \\
\hline Eurytoma & Eurytomidae & 0.00 & 1.00 & 0.00 & 0.00 & 1.00 & 1.00 & 0.00 & 0.00 & 0.00 & 0.00 & 0.00 \\
\hline B. oxymora & Ichneumonidae & 0.00 & 0.00 & 0.00 & 1.00 & 0.00 & 0.00 & 0.00 & 0.00 & 0.00 & 0.00 & 0.00 \\
\hline G. bunoh & Ichneumonidae & 0.00 & 0.00 & 1.00 & 0.00 & 1.00 & 1.00 & 0.00 & 0.00 & 0.00 & 0.00 & 0.00 \\
\hline Ichneumonid & Ichneumonidae & 0.00 & 1.00 & 1.00 & 2.00 & 0.00 & 0.00 & 0.00 & 0.00 & 0.00 & 0.00 & 0.00 \\
\hline P. varius & Ichneumonidae & 0.00 & 0.00 & 2.00 & 0.00 & 0.00 & 0.00 & 0.00 & 1.00 & 0.00 & 1.00 & 1.00 \\
\hline Xanthopimpla & Ichneumonidae & 0.00 & 0.00 & 0.00 & 1.00 & 0.00 & 0.00 & 0.00 & 0.00 & 0.00 & 0.00 & 0.00 \\
\hline C. picticeps & Reduviidae & 1.00 & 1.00 & 2.00 & 3.00 & 2.00 & 2.00 & 2.00 & 2.00 & 2.00 & 2.00 & 1.00 \\
\hline S. dichotomus & Reduviidae & 0.00 & 0.00 & 0.00 & 0.00 & 2.00 & 0.00 & 0.00 & 0.00 & 0.00 & 0.00 & 0.00 \\
\hline
\end{tabular}

TABLE 2. DISTRIBUTION OF RESIDENT NATURAL ENEMIES (sweep net) ALONG VARIOUS LIGHT INTENSITIES

\begin{tabular}{|c|c|c|c|c|c|c|c|c|c|}
\hline \multirow[b]{3}{*}{ Species } & \multirow[b]{3}{*}{ Family } & \multicolumn{8}{|c|}{ No. of individuals captured by sweep net } \\
\hline & & \multicolumn{8}{|c|}{ Light intensity (fc) } \\
\hline & & $\begin{array}{l}1000- \\
1999\end{array}$ & $\begin{array}{c}2000- \\
2999\end{array}$ & $\begin{array}{c}3000- \\
3999\end{array}$ & $\begin{array}{c}5000- \\
5999\end{array}$ & $\begin{array}{c}6000- \\
6999\end{array}$ & $\begin{array}{l}7000- \\
7999\end{array}$ & $\begin{array}{c}8000- \\
8999\end{array}$ & $\begin{array}{l}9000- \\
9999\end{array}$ \\
\hline A. alluela & Braconidae & 3 & 0 & 0 & 0 & 0 & 0 & 0 & 0 \\
\hline A. psychidivorus & Braconidae & 0 & 0 & 0 & 0 & 0 & 1 & 0 & 0 \\
\hline D. metesae & Braconidae & 4 & 0 & 0 & 0 & 0 & 0 & 0 & 0 \\
\hline B. carinata & Chalcididae & 0 & 0 & 0 & 0 & 0 & 0 & 2 & 3 \\
\hline B. lugubris & Chalcididae & 4 & 1 & 0 & 0 & 1 & 0 & 4 & 3 \\
\hline Sympiesis & Eulophidae & 1 & 0 & 0 & 0 & 0 & 0 & 2 & 0 \\
\hline Tetrastichus & Eulophidae & 3 & 1 & 0 & 0 & 0 & 0 & 0 & 0 \\
\hline E. catoxanthae & Eupelmidae & 1 & 0 & 0 & 1 & 0 & 0 & 0 & 0 \\
\hline Eurytoma & Eurytomidae & 2 & 2 & 0 & 0 & 3 & 1 & 1 & 2 \\
\hline B. oxymora & Ichneumonidae & 2 & 0 & 0 & 0 & 0 & 0 & 0 & 0 \\
\hline C. picticeps & Reduviidae & 3 & 0 & 4 & 0 & 0 & 1 & 1 & 3 \\
\hline
\end{tabular}

(1000-1999 fc), possibly indicating less active at these range of light intensities (Table 2). However, further analysis indicates that light intensity has no significant effect on the resident population (Table 3).

Insect activities in relation to relative humidity $(\boldsymbol{R H})$. With sticky traps, the activities of the insect natural enemies were observed higher at medium humidity levels of between $60 \%-79 \%$ RH (Table
4). These include all three species of braconids (A. aluella, D. metesae and S. spinator), chalcids (B. carinata), most eulophids (P. imbreus, Sympiesis and Tetrastichus) and ichneumonids (G. bunoh and $P$. varius). Insect activities are shown to be significantly higher $(\mathrm{p}<0.05)$ at the medium range of humidity levels (50-69\%) (Table 6).

Insects tend to rest at the ground covers at higher humidity levels; hence, more individuals were 
TABLE 3. MEAN NUMBER OF INSECTS CAPTURED PER INSECT SPECIES UNDER VARIED LIGHT INTENSITIES

\begin{tabular}{lccc}
\hline & \multicolumn{3}{c}{ Mean number of insects captured per species } \\
\cline { 2 - 4 } & $\begin{array}{c}\text { Low } \\
(300-3999 \mathrm{fc})\end{array}$ & $\begin{array}{c}\text { Medium } \\
(4000-7999 \mathrm{fc})\end{array}$ & $\begin{array}{c}\text { High } \\
(8000-10000 \mathrm{fc})\end{array}$ \\
\hline $\begin{array}{l}\text { Activities of insect (sticky } \\
\text { trap captures) }\end{array}$ & $2.55^{\mathrm{a}}$ & $2.70^{\mathrm{a}}$ & $0.50^{\mathrm{b}}$ \\
$\begin{array}{l}\text { Resident population (sweep net } \\
\text { captures) }\end{array}$ & $2.80^{\mathrm{a}}$ & & $1.90^{\mathrm{a}}$ \\
\hline
\end{tabular}

Note: Means with the same letter within the same row are not significantly different (ANOVA, Wilcoxon Mann-Whitney, $\mathrm{p}=0.05)$.

TABLE 4. DISTRIBUTION OF NATURAL ENEMIES' ACTIVITY (sticky trap) ALONG VARIOUS RELATIVE HUMIDITIES

\begin{tabular}{|c|c|c|c|c|c|c|c|}
\hline \multirow[b]{3}{*}{ Species } & \multirow[b]{3}{*}{ Family } & \multicolumn{6}{|c|}{ No. of individuals captured by sticky traps } \\
\hline & & \multicolumn{6}{|c|}{ Relative humidity (\%) } \\
\hline & & $30-39$ & $40-49$ & $50-59$ & $60-69$ & $70-79$ & $80-89$ \\
\hline A. aluella & Braconidae & 0.00 & 0.00 & 4.00 & 13.00 & 9.00 & 1.00 \\
\hline D. metesae & Braconidae & 0.00 & 0.00 & 0.00 & 5.00 & 3.00 & 1.00 \\
\hline S. spinator & Braconidae & 0.00 & 0.00 & 0.00 & 4.00 & 2.00 & 0.00 \\
\hline A. thylax & Ceraphronidae & 0.00 & 0.00 & 2.00 & 3.00 & 1.00 & 0.00 \\
\hline B. lugubris & Chalcididae & 0.00 & 0.00 & 0.00 & 1.00 & 1.00 & 0.00 \\
\hline B. carinata & Chalcididae & 0.00 & 1.00 & 0.00 & 2.00 & 0.00 & 0.00 \\
\hline C. arcufer & Cleridae & 0.00 & 0.00 & 0.00 & 2.00 & 2.00 & 0.00 \\
\hline P. imbreus & Eulophidae & 0.00 & 1.00 & 0.00 & 4.00 & 3.00 & 0.00 \\
\hline P. anomalus & Eulophidae & 0.00 & 0.00 & 2.00 & 1.00 & 2.00 & 0.00 \\
\hline Sympiesis & Eulophidae & 0.00 & 1.00 & 0.00 & 2.00 & 1.00 & 0.00 \\
\hline Tetrastichus & Eulophidae & 0.00 & 0.00 & 0.00 & 2.00 & 1.00 & 0.00 \\
\hline E. catoxanthae & Eupelmidae & 0.00 & 0.00 & 0.00 & 1.00 & 1.00 & 1.00 \\
\hline Eurytoma & Eurytomidae & 0.00 & 1.00 & 0.00 & 1.00 & 0.00 & 1.00 \\
\hline B. oxymora & Ichneumonidae & 0.00 & 0.00 & 0.00 & 0.00 & 1.00 & 0.00 \\
\hline G. bunoh & Ichneumonidae & 0.00 & 0.00 & 0.00 & 3.00 & 0.00 & 0.00 \\
\hline Ichneumonid & Ichneumonidae & 0.00 & 0.00 & 0.00 & 1.00 & 3.00 & 0.00 \\
\hline P. varius & Ichneumonidae & 0.00 & 0.00 & 0.00 & 3.00 & 2.00 & 0.00 \\
\hline Xanthopimpla & Ichneumonidae & 0.00 & 0.00 & 0.00 & 1.00 & 0.00 & 0.00 \\
\hline S. dichotomus & Reduviidae & 0.00 & 0.00 & 0.00 & 1.00 & 1.00 & 0.00 \\
\hline C. picticeps & Reduviidae & 1.00 & 2.00 & 4.00 & 12.00 & 3.00 & 0.00 \\
\hline
\end{tabular}

captured by sweep nets. Braconids like A. aluella and D. metesae seem to be resting at very high humidities of between $80 \%-90 \%$ RH (Table 5). However, chalcid species like B. carinata and B. lugubris appeared resting between 60\%-70\% RH. Eulophids like Sympiesis seem to be resting at medium (50\%-60\% $\mathrm{RH})$ humidities, while Tetrastichus, at higher (70\%$80 \% \mathrm{RH}$ ) humidities (Table 5). It was also confirmed that significantly higher $(\mathrm{p}<0.05)$ insect population are resting at the highest range of humidity $(70 \%$ $89 \%$ ) (Table 6).

Similarly, the abundance of predators (i.e. C. picticeps) in sticky traps indicated higher activities between $60 \%-70 \% \mathrm{RH}$ and consequently, most of them were also caught by sweep net, at the same humidity levels (Table 5).

Insect activities in relation to temperature $\left({ }^{\circ} \mathrm{C}\right)$. In terms of natural enemies captured by sticky traps, a majority of the insect families were found more active between $30^{\circ} \mathrm{C}-34^{\circ} \mathrm{C}$, with the exceptions of chalcids (B. carinata) and reduviid (C. picticeps), being more active at the higher range of $35^{\circ} \mathrm{C}-39^{\circ} \mathrm{C}$ (Table 7). In general, significantly lower $(p<0.05)$ activities occurred at both the lower or higher temperatures from this range $\left(30^{\circ} \mathrm{C}-34^{\circ} \mathrm{C}\right)$ (Table 9). 
TABLE 5. DISTRIBUTION OF RESIDENT NATURAL ENEMIES (sweep net) ALONG VARIOUS RELATIVE HUMIDITIES

\begin{tabular}{|c|c|c|c|c|c|c|c|}
\hline \multirow[b]{3}{*}{ Species } & \multirow[b]{3}{*}{ Family } & \multicolumn{6}{|c|}{ No. of individuals captured by sweep net } \\
\hline & & \multicolumn{6}{|c|}{ Relative humidity $(\%)$} \\
\hline & & $40-49$ & $50-59$ & $60-69$ & $70-79$ & $80-89$ & $90-99$ \\
\hline A. psychidivorus & Braconidae & 0.00 & 0.00 & 1.00 & 0.00 & 0.00 & 0.00 \\
\hline A. alluela & Braconidae & 0.00 & 0.00 & 0.00 & 0.00 & 3.00 & 0.00 \\
\hline D. metesae & Braconidae & 0.00 & 0.00 & 0.00 & 2.00 & 2.00 & 0.00 \\
\hline B. carinata & Chalcididae & 0.00 & 1.00 & 4.00 & 0.00 & 0.00 & 0.00 \\
\hline B. lugubris & Chalcididae & 0.00 & 3.00 & 4.00 & 3.00 & 3.00 & 1.00 \\
\hline Sympiesis & Eulophidae & 0.00 & 2.00 & 1.00 & 1.00 & 0.00 & 0.00 \\
\hline Tetrastichus & Eulophidae & 1.00 & 0.00 & 0.00 & 2.00 & 1.00 & 0.00 \\
\hline E. catoxanthae & Eupelmidae & 0.00 & 0.00 & 0.00 & 0.00 & 1.00 & 0.00 \\
\hline Eurytoma & Eurytomidae & 1.00 & 2.00 & 1.00 & 5.00 & 2.00 & 0.00 \\
\hline B. oxymora & Ichneumonidae & 0.00 & 0.00 & 0.00 & 1.00 & 0.00 & 1.00 \\
\hline C. picticeps & Reduviidae & 0.00 & 1.00 & 3.00 & 6.00 & 1.00 & 1.00 \\
\hline
\end{tabular}

TABLE 6. MEAN NUMBER OF INSECTS CAPTURED PER INSECT SPECIES UNDER VARIED RELATIVE HUMIDITIES

\begin{tabular}{lccc}
\hline & \multicolumn{3}{c}{ Mean number of insects captured per species } \\
\cline { 2 - 3 } & Low (30\%-49\%) & Medium (50\%-69\%) & High (70\%-89\%) \\
\hline $\begin{array}{l}\text { Activities of insect } \\
\text { (sticky trap captures) }\end{array}$ & $0.35^{\mathrm{a}}$ & $3.70^{\mathrm{b}}$ & $2.00^{\mathrm{ab}}$ \\
$\begin{array}{l}\text { Resident population } \\
\text { (sweep net captures) }\end{array}$ & $0.20^{\mathrm{a}}$ & $2.10^{\mathrm{ab}}$ & $3.30^{\mathrm{b}}$ \\
\hline
\end{tabular}

Note: Means with the same letter within the same row are not significantly different (ANOVA, Wilcoxon Mann-Whitney, $\mathrm{p}=0.05)$.

TABLE 7. DISTRIBUTION OF NATURAL ENEMIES' ACTIVITY (sticky trap) ALONG VARIOUS TEMPERATURES

\begin{tabular}{|c|c|c|c|c|c|}
\hline \multirow[b]{3}{*}{ Species } & \multirow[b]{3}{*}{ Family } & \multicolumn{4}{|c|}{ No. of individuals captured by sticky traps } \\
\hline & & \multicolumn{4}{|c|}{ Temperature $\left({ }^{\circ} \mathrm{C}\right)$} \\
\hline & & $25-29$ & $30-34$ & $35-39$ & $40-44$ \\
\hline A. aluella & Braconidae & 6.00 & 15.00 & 4.00 & 0.00 \\
\hline D. metesae & Braconidae & 1.00 & 7.00 & 1.00 & 0.00 \\
\hline S. spinator & Braconidae & 0.00 & 5.00 & 1.00 & 0.00 \\
\hline A. thylax & Ceraphronidae & 0.00 & 4.00 & 1.00 & 0.00 \\
\hline B. lugubris & Chalcididae & 1.00 & 1.00 & 0.00 & 0.00 \\
\hline B. carinata & Chalcididae & 0.00 & 1.00 & 2.00 & 0.00 \\
\hline C. arcufer & Cleridae & 0.00 & 3.00 & 1.00 & 0.00 \\
\hline P. imbreus & Eulophidae & 0.00 & 8.00 & 0.00 & 0.00 \\
\hline P. anomalus & Eulophidae & 1.00 & 3.00 & 0.00 & 0.00 \\
\hline Sympiesis & Eulophidae & 0.00 & 4.00 & 0.00 & 0.00 \\
\hline Tetrastichus & Eulophidae & 0.00 & 2.00 & 1.00 & 0.00 \\
\hline E. catoxanthae & Eupelmidae & 1.00 & 1.00 & 1.00 & 0.00 \\
\hline Eurytoma & Eurytomidae & 1.00 & 2.00 & 0.00 & 0.00 \\
\hline B. oxymora & Ichneumonidae & 0.00 & 1.00 & 0.00 & 0.00 \\
\hline G. bunoh & Ichneumonidae & 0.00 & 3.00 & 0.00 & 0.00 \\
\hline Ichneumonid & Ichneumonidae & 1.00 & 3.00 & 0.00 & 0.00 \\
\hline P. varius & Ichneumonidae & 0.00 & 3.00 & 2.00 & 0.00 \\
\hline Xanthopimpla & Ichneumonidae & 0.00 & 1.00 & 0.00 & 0.00 \\
\hline C. picticeps & Reduviidae & 0.00 & 9.00 & 10.00 & 1.00 \\
\hline S. dichotomus & Reduviidae & 0.00 & 2.00 & 0.00 & 0.00 \\
\hline
\end{tabular}


TABLE 8. DISTRIBUTION OF RESIDENT NATURAL ENEMIES (sweep net) ALONG VARIOUS TEMPERATURES

\begin{tabular}{|c|c|c|c|c|}
\hline \multirow[b]{3}{*}{ Species } & \multirow[b]{3}{*}{ Family } & \multicolumn{3}{|c|}{ No. of individuals captured by sweep net } \\
\hline & & \multicolumn{3}{|c|}{ Temperature $\left({ }^{\circ} \mathrm{C}\right)$} \\
\hline & & $25-30$ & $31-34$ & $35-40$ \\
\hline A psychidivorus & Braconidae & 0.00 & 1.00 & 0.00 \\
\hline A. alluela & Braconidae & 3.00 & 0.00 & 0.00 \\
\hline D. metesae & Braconidae & 3.00 & 1.00 & 0.00 \\
\hline B. carinata & Chalcididae & 0.00 & 0.00 & 5.00 \\
\hline B. lugubris & Chalcididae & 5.00 & 2.00 & 7.00 \\
\hline Sympiesis & Eulophidae & 1.00 & 1.00 & 2.00 \\
\hline Tetrastichus & Eulophidae & 3.00 & 1.00 & 0.00 \\
\hline E. catoxanthae & Eupelmidae & 1.00 & 0.00 & 0.00 \\
\hline Eurytoma & Eurytomidae & 2.00 & 5.00 & 4.00 \\
\hline B. oxymora & Ichneumonidae & 1.00 & 1.00 & 0.00 \\
\hline C. picticeps & Reduviidae & 3.00 & 5.00 & 4.00 \\
\hline
\end{tabular}

TABLE 9. MEAN NUMBER OF INSECTS CAPTURED PER INSECT SPECIES UNDER VARIED TEMPERATURES

\begin{tabular}{|c|c|c|c|}
\hline & \multicolumn{3}{|c|}{ Mean number of insects captured per species } \\
\hline & Low $\left(25^{\circ} \mathrm{C}-29^{\circ} \mathrm{C}\right)$ & Medium $\left(30^{\circ} \mathrm{C}-34^{\circ} \mathrm{C}\right)$ & High $\left(35^{\circ} \mathrm{C}-39^{\circ} \mathrm{C}\right)$ \\
\hline $\begin{array}{l}\text { Activities of insect } \\
\text { (sticky trap captures) }\end{array}$ & $0.60^{\mathrm{a}}$ & $3.90^{\mathrm{b}}$ & $1.20^{\mathrm{a}}$ \\
\hline $\begin{array}{l}\text { Resident population } \\
\text { (sweep net captures) }\end{array}$ & $2.00^{\mathrm{a}}$ & $1.50^{\mathrm{a}}$ & $2.00^{\mathrm{a}}$ \\
\hline
\end{tabular}

Note: Means with the same letter within the same row are not significantly different (ANOVA, Wilcoxon Mann-Whitney, $\mathrm{p}=0.05)$.

Braconids (A. aluella, D. metesae) and the eulophid (Tetrastichus) appeared resting at lower temperatures between $25^{\circ} \mathrm{C}-30^{\circ} \mathrm{C}$. Eurytomids (Eurytoma sp.) and reduviid (C. picticeps) appeared resting between $31^{\circ} \mathrm{C}-34^{\circ} \mathrm{C}$ while chalcids (B. carinata, B. lugubris) and eulophid (Sympiesis sp.) resting at higher temperatures of between $35^{\circ} \mathrm{C}$ $-39^{\circ} \mathrm{C}$ (Table 8).

In general, temperature seems to have no direct effect on the resident population ( $\mathrm{p}>0.05)$. However as mentioned above, there were some indications that more insects were resting at both the cooler $\left(25^{\circ} \mathrm{C}-29^{\circ} \mathrm{C}\right)$ and hotter $\left(35^{\circ} \mathrm{C}-39^{\circ} \mathrm{C}\right)$ temperatures (Table 9).

\section{DISCUSSION}

In this current study, the insect natural enemies within the bagworm infested plots were surveyed by sticky traps and sweep nets. The activities of natural enemies can be estimated based on captures of sticky traps as reported by Dreistadt (1998), Idris and Grafius (1998) and Gray (2009), whereas resident insect population can be assumed based on the captures of sweep net as conducted by UC IPM (2010) and Peña et al. (2010).

It was shown from this study that sticky traps consistently captured a higher species density of natural enemies compared to sweep net. This is logical, as the traps were left for two consecutive days, compared to sweep net sampling which was periodically done from morning to afternoon. However, for comparison between the two methods, the mean number for each species was calculated.

It was observed that species diversity of the hymenopterous parasitoids and predators were relatively higher on the $C$. cobanensis plant (ShannonWiener index, 2.32), compared to the ground covers of the non-harvesting paths (Shannon-Wiener indices between 1.10-1.12) (Figures 1 to 3). The hymenopterous parasitoids, which consisted of the families Ichneumonidae, Eulophidae, Chalcididae, Braconidae, Eurytomidae, Eupelmidae and Ceraphronidae have all been recorded as important parasitoids for the bagworms (Norman et al., 1996) including one braconid species important for nettle caterpillars (Norman et al., 1998). Apart from these parasitoids, two common species of predators (Hemiptera: Reduviidae) were commonly found 
as general predators for bagworms and nettle caterpillars (Norman et al., 1998). It is also necessary from this study to determine any different types of natural enemies emerging from Pteroma pendula, so as to complement the earlier studies (Norman et al., 1996; 1998).

The results showed that within the $C$. cobanensis plant, Chalcididae (B. lugubris, B. lasus and B. carinata) was more dominant than Braconidae (D. metesae and A. aluella), and that these two families were more dominant than the other families of Hymenopteran parasitoids (Figure 1). This also supports the findings of Naganuma and Hespenheide (1988), which reported on these two predominantly parasitic wasps families visiting the extra floral nectaries of Baccharis sarothroides Gray (desert broom). It was also reported by that chalcids eventually 'kicked' the braconids away (Naganuma and Hespenheide, 1988).

On the ground covers within the oil palm planting block, there were lower species diversity (Shannon-Wiener index, 1.10), and lower density of insect natural enemies (Figure 2). The families of parasitoids recorded were Ichneumonidae, Eulophidae, Chalcididae, Eurytomidae and Braconidae. Eurytoma sp. appeared to be captured more frequently than the others, with no indications of dominance by neither chalcids nor braconids. This possibly indicates that the food sources for these parasitoids were scarce or not available, because the composition of ground covers in the survey area consisted mainly of ferns (e.g. Nephrolepis biserrata, Stenochlaena palustris). It was also important to note that within this area, the reduuvid predator, $C$. picticeps and a clerid predator, Callimerus arcufer were commonly captured, indicating that these two predators preferred to dwell on the ground covering fern species. Heteroptera (Miridae, Reduviidae) has been recorded dwelling on ferns (Wheeler, 2001; Ballentes, et al., 2006). S. dichotomus, on the other hand, was only captured among the C. cobanensis plant and was not detected in the oil palm planting block. This may indicate the dependency of $S$. dichotomus on the beneficial plant.

Similarly, the low captures of insect parasitoids (less than five individuals per species) within the oil palm planting block, indicates the important and contributory effects of $C$. cobanensis plant as nectar provider to the population of parasitoids. In the absence of this beneficial plant, only three species of parasitoids appeared to be frequently captured, with the reduviid predator gaining dominance (Figure 3). As expected, the ShannonWiener index of diversity within the oil palm planting block (1.12) was 50\% lower than on the $C$. cobanensis plant (2.32).

Using sticky traps, the activities of the insect natural enemies was highest at light intensities of between 2000-4999 fc, with the highest activity recorded between 4000-4999 fc. In contrast, sweep net sampling showed high capture of the natural enemies at the lower range of light intensity (1000-1999 fc). These results suggest that most insect natural enemies are active at medium light intensities and likely to be resting at lower range of light intensities.

The intensity, duration and quality of light have an important influence on the biology and behaviour of most insects (Jervis, 2005). High light intensity seems to increase the general activity of diurnal predators and parasitoids (Jervis et al., 2005). For example, the flight and locomotory movements of the Gypsy moth's pupal parasitoid, Brachymeria intermedia (Hymenoptera: Chalcididae) increased as temperature and light intensity increased. Significant peaks in activity also occurred during the photo phase (Barbosa and Frongillo, 1977). For Aphelinus asychis (Hymenoptera: Aphelinidae), light intensity modified its biological and ecological traits. High light intensity resulted in a shorter developmental duration, higher incidence of females and longer life span of the female parasitoid (Sengonca et al., 2008). However, it was reported that this parasitoid can also completely develop and reproduce at low light intensities of 100 and $700 \mathrm{fc}$.

In general, several species of hymenopteran families (braconid, ichneumonids and chalcids) were found active between 2000-4000 fc. In this study although ichneumonids were found active over a broad range of light intensities (1000$13000 \mathrm{fc}), P$. varius and S. spinator seem to be active in less intense sunlight, between 2000-4000 fc. This supported the findings of Idris and Grafius (1998), which showed that the ichneumonid, Diadegma insulare is active in the early morning (0800-1000) and peaked by noon.

In contrast, several species of eulophids were active at higher light intensities between 4000-5000 fc. Eulophids are considered mainly hyperparasitoids (Norman et al., 1996), and therefore it is hypothesised that this could be a strategy for these parasitoids to be active at a later part of the day (with higher light intensity), in order to successfully parasitise the primary parasitoids (braconids, ichneumonids and chalcids), which have been actively ovipositing during the earlier part of the day. This current study also supported the previous report by Idoine and Ferro (1990) which indicated that the egg parasitoid eulophid, Edovum puttleri are active at around mid-day to the afternoon, i.e. between 6-11 hr after sunrise.

The braconid, D. metesae, on the other hand, appeared to have periodic activities between 300$1000 \mathrm{fc}, 3000-4000 \mathrm{fc}$ and 6000-7000 fc. These periodic activities in different parts of the day, could be a parasitism strategy, in the presence of the competing hyperparasitoids (eulophids). 
In general, almost all parasitoids seem to be resting in much lower light intensities (1000-2000 fc). However, some chalcids rest in extreme intense sunlight of more than $8000 \mathrm{fc}$. Kamm (1990) reported that the alfafa seed chalcids, Bruchophagous roddi (Hymenoptera: Superfamily Chalcidoidea, Family Eurytomidae) displayed no preference for baited targets at noon (1200 hr). Preference or activity was not shown at 1-2 hr after sunrise or 1-2 hr before sunset. Kamm (1990) hypothesised that chalcid response/activity was controlled by the polarisation characteristics of sky light. This could probably explain why parasitoids are resting in the early part of morning when the light intensity is low.

Light intensity can also modify biological and ecological traits of insects. For example, high light intensity resulted in a shorter developmental duration, higher incidence of females and longer life span of the female Aphelinus asychis (Hymenoptera: Aphelinidae) (Schirmer et al., 2008). Aphelinids like Encarsia formosa and Eretmocerus eremicus parasitised approximately twice as many whitefly hosts at the high light intensity (112-114 $\mathrm{W} \mathrm{m}^{-2}$ ) and long day length (L 16:D $8 \mathrm{hr}$ ) treatment than at the low light intensity (12-14 $\mathrm{W} \mathrm{m}^{-2}$ ) and short daylength (L 8:D $16 \mathrm{hr}$ ) treatment (Zilahi-Balogh et al., 2006). In the oil palm environment, it was observed that parasitoids were more active at the low to medium range of light intensities, and rest during very high light intensities.

Predators also appeared to be active over a broad range of light intensities from 300-13 $000 \mathrm{fc}$ but $C$. picticeps was more active between $3000-4000$ $\mathrm{fc}$, which is also within the active range among the parasitoids.

Using sticky traps, the activities of most insect natural enemies was observed high at humidities of between $60 \%-79 \%$ RH. Using sweep net, the captures was also high between 70\%-89\% RH. The parasitoid for Aphis gossipyi, Aphelinus asychis (Hymenoptera: Aphelinidae) can completely develop and reproduce at relative humidities of $30 \%$ and $60 \%$ (Sengonca et. al., 2008).

Most of the parasitoids seem to be more active at medium humidity levels of between $60-70 \mathrm{RH}$, as reflected by the high activity of most insect parasitoids within that humidity levels. These include braconids, chalcids, most eulophids and ichneumonids. Moisture also exerts an influence on the growth and survival of insects. Some species become dormant in the absence of adequate moisture as in the case of the eulophid parasitoid Sympiesis hyblaea of the teak defoliator Hyblaea puera (Nair, 2007). Parasitism of Trichogramma chilonis (Hymenoptera: Trichogrammatidae) was good when bred at RH of $70 \%-80 \%$. In the laboratory, most species are active $40 \%-60 \% \mathrm{RH}$, with lower threshold: $25 \% \mathrm{RH}$ and higher, $70 \% \mathrm{RH}$. In India, low $\mathrm{RH}(9 \%-22 \%)$ is detrimental to the parasitoid.
(Upadhyay et al., 2001). On the other hand, sawflies (Hymenoptera: Pamphiliidae) tend to be more active in low RH of $20 \%$ (Holusa and Kuras, 2010).

Insects tend to rest at the ground covers, at higher humidity levels, hence more were captured by sweep nets. Braconids seem to be resting at very high humidities of between $80 \%-90 \% \mathrm{RH}$. This could be the fact that it was very early in the morning and the light intensity was too low for their flight activities. However, other parasitoids like eulophids and chalcids were also caught by sweep nets, between 50\%-80\% RH when most parasitoids are likely to be active. This may suggest that these parasitoids were in abundance and some may be residing at the ground covers. Similarly, the abundance of predators (i.e. C. picticeps), which are also highly active between 60-70 $\mathrm{RH}$, were consequently caught by sweep net.

In relation to temperature, almost all the insect natural enemies in this study were active at moderately high temperatures of between $30^{\circ} \mathrm{C}-34^{\circ} \mathrm{C}$. There are however some exceptions like chalcids (B. carinata) and reduviid (C. picticeps) being more active at even higher temperatures of $35^{\circ} \mathrm{C}-39^{\circ} \mathrm{C}$. In a temperate country (Spain), it was reported that ichneumonids actively fly at cool temperatures of $25^{\circ} \mathrm{C}-27^{\circ} \mathrm{C}$ during summer (Mazon et al., 2009).

The predatory flower bug Orius similis Zheng (Heteroptera: Anthocoridae) has a decreased duration of embryonic and nymphal development as well as total percentage mortality during the development with increasing temperature between $18^{\circ} \mathrm{C}$ and $30^{\circ} \mathrm{C}$ (Ahmadi et al., 2007). The daily oviposition ranged from $1.0\left(18^{\circ} \mathrm{C}\right)$ to $6.9\left(30^{\circ} \mathrm{C}\right)$ eggs per day with A. gossypii and M. persicae as prey, respectively (Ahmadi et al., 2007). Based from the field captures in this current study, the predator, C. picticeps seem to be more active at temperatures of between $35^{\circ} \mathrm{C}-39^{\circ} \mathrm{C}$. This increase in the predator's activity might be related to oviposition.

Using sweep net, most insect parasitoids were collected between $25^{\circ} \mathrm{C}-30^{\circ} \mathrm{C}$, indicating many species (some braconids and eulophids) were not very active at this lower temperature range. Eurytomid could be in abundance as most parasitoids were found active between $31^{\circ} \mathrm{C}-34^{\circ} \mathrm{C}$. Some chalcids and eulophids could also be in abundance as they were mostly caught by sweep net within temperatures of between $35^{\circ} \mathrm{C}-40^{\circ} \mathrm{C}$.

Temperature had a significant effect on the developmental duration of the aphid parasitoid, A. asychis as well as on the percentage and longevity of females, while mortality from mummification to emergence, fecundity and host feeding were only slightly affected (Sengonca et al., 2008). This parasitoid can successfully develop and reproduce between $18^{\circ} \mathrm{C}-30^{\circ} \mathrm{C}$ (Sengonca et al., 2008). Parasitism of T. chilonis was good when 
bred at $25^{\circ} \mathrm{C}-28.3^{\circ} \mathrm{C}$. Most species were active and fecund at $20^{\circ} \mathrm{C}-29^{\circ} \mathrm{C}$, with lower threshold: $9^{\circ} \mathrm{C}$ and higher, $36^{\circ} \mathrm{C}$. Parasitism of Trichogramma increased with temperature, reaching a maximum at $20^{\circ} \mathrm{C}-25^{\circ} \mathrm{C}$, with more parasitism at higher than lower temperatures (Pavlik, 1993). Very high temperatures $\left(43^{\circ} \mathrm{C}-47^{\circ} \mathrm{C}\right)$ were detrimental to parasitoids (Upadhyay et al., 2001).

\section{CONCLUSION}

This study has shown that the overall diversity of insect natural enemies occurring on the C. cobanensis plant was higher compared to the harvesting paths in the oil palm planting block. This suggests the importance of $C$. cobanensis in establishing populations of the bagworm natural enemies, especially the hymenopterous parasitoids, which are potent biocontrol agent for the bagworms. Therefore, it is recommended that the $C$. cobanensis plant should also be planted within the oil palm block, wherever possible, in areas with adequate sunlight, in addition to planting at the roadside. Overall, there are significant increase in natural enemies activities at low to medium light intensities $(<8000 \mathrm{fc})$, medium humidity levels (50\%-69\%) and medium temperatures $\left(30^{\circ} \mathrm{C}-34^{\circ} \mathrm{C}\right)$. This information would provide better understanding on the environment suited and conducive for the diversity and activities of these natural enemies for a long-term, sustainable, control of bagworms.

\section{ACKNOWLEDGEMENT}

The authors thank the Director-General of MPOB for permission to publish this article. A round of thanks for the staff of the Entomology Laboratory 1, Biological Research Division, MPOB, particularly Hajijah Shamsuddin, Zuriahti Khamis, Norhassan Mohd Yob, Tunku Akhirudin Aris and Mat Tahir Bung Ruziz, for their help and hard work in conducting sampling, field experiments and data input.

\section{REFERENCES}

AHMADI, K; SENGONCA, $\mathrm{C}$ and BLAESER, $\mathrm{P}$ (2007). Effect of two different temperatures on the biology of predatory flower bug Orius similis Zheng (Heteroptera: Anthocoridae) with two different aphid species as prey. Türk. Entomology. Derg., 31(4): 253-268.

BARBOSA, $\mathrm{P}$ and FRONGILLO, E A (1977). Influence of light intensity and temperature on the locomotory and flight activity of Brachymeria intermedia (Hymenoptera: Chalcididae) a pupal parasitoid of the gypsy moth. BioControl, 22(4): 405-411.

BASRI, M W (1993). Life History, Ecology and Economic Impact of the Bagworm, Metisa plana Walker (Lepidoptera: Psychidae) on the Oil Palm Elaeis guineensis Jacquin (Palmae) in Malaysia. Ph.D. thesis, University of Guelph. p. 231.

BASRI, M W; SIMON, S; RAVIGADEVI, $S$ and OTHMAN, A (1999). Beneficial plants for the natural enemies of the bagworm in oil palm plantations. Proc. of the 1999 PORIM International Palm Oil Congress - Emerging Technologies and Opportunities in the Next Millennium (Ariffin, D; Chan, $\mathrm{K}$ W and Sharifah, S R S A eds.). PORIM, Bangi. p. 441-455.

BALLENTES, M G; MOHAGAN, A B; GAPUD, V P; ESPALLARDO, M C P and ZARCILLA, M O (2006). Arthropod faunal diversity and relevant interrelationships of critical resources in $\mathrm{Mt}$. Malindang, Misamis Occidental. Biodiversity Research Programme for Development in Mindanao: Focus on Mt. Malindang and Environs. Southeast Asian Regional Center for Graduate Study and Research in Agriculture (SEARCA). 166 pp.

DREISTADT, S H (1998). Sticky Trap Monitoring of Insect Pests. ANR Publications. 8 pp.

GRAY, B (2009). Observations on insect flight in a tropical forest plantation. Flight activity of Buprestidae and Othniidae (Coleoptera). J. Applied Entomology, 76 (1-4): 190-195.

HOLUSA, J and KURAS, T (2010) Diurnal behaviour of Cephalcia lariciphila (Hymenoptera: Pamphiliidae): relation to climatic factors and significance for monitoring. Eur J Forest Res 129: 243 248.

HO, C T; KHOO, K C; YUSOF, I and DZOLKIFLI, O (2003). Comparative studies on the use of beneficial plants for natural suppression of bagworm infestation in oil palm. Proc. of the 2003 PIPOC International Palm Oil Congress - Agriculture Conference. p. 372-424.

IDRIS, A B and GRAFIUS, E (1998). Diurnal flight activity of Diadegma insulare (Hymenoptera: Ichneumonidae), a parasitoid of the diamondback moth (Lepidoptera: Plutellidae), in the field. Environmental Entomology Vol. 27 No. 2: 406-414.

IDOINE, K and FERRO, D N (1990). Diurnal timing of ovipositional activities of Edovum puttleri (Hymenoptera: Eulophidae), an egg parasitoid of Leptinotarsa decemlineata (Coleoptera: 
Chrysomelidae). Environmental Entomology Vol. 19 No. 1: 104-107.

JERVIS, M A (2005). Insects as Natural Enemies: A Practical Perspective. Springer. 748 pp.

JERVIS, M A; COPLAND, M J W and HARVEY, J A (2005). The life cycle. Insects as Natural Enemies: A Practical Perspective (Jarvis, M ed.). Springer. 748 pp.

KAMM, J A (1990). Control of olfactory-included behavior in alfalfa seed chalcid (Hymenoptera: Eurytomidae) by celestial light. J. Chemical Ecology, 16(2): 290-300.

MAZON, MARINA; BORDERA, SANTIAGO and RODRIGUEZ-BERRIO, ALEXANDER (2009). Diurnal flight activity of Ichneumonidae (Insecta: Hymenoptera) in Cabaneros National Park (Spain). J. Natural History, Vol. 43 No. 22: 21-22.

NAIR, KS S (2007). Tropical Forest Insect Pests: Ecology, Impact and Management. Cambridge University Press. 404 pp.

NAGANUMA, K and HESPENHEIDE, H A (1988). Behaviour of visitors at insect produced analogues of extra floral nectaries on Baccharis sarothroides Gray. Southwestern Naturalist, 33(3): 275-286.

NORMAN, K; WALKER, A K; MOHD BASRI, W; LASALLE, J and POLASZEK, A (1996). Hymenopterous parasitoids of the bagworm, Metisa plana and Mahasena corbetti on oil palm in Peninsular Malaysia. Bulletin of Entomological Research, 86: 423439.

NORMAN, H K; BASRI, M W and ZULKEFLI, M (1998). Handbook of Common Parasitoids and Predators Associated with Bagworms and Nettle Caterpillars in Oil Palm Plantations. PORIM, Bangi. 29 pp.

NOR SARASHIMATUN, S; TEH, C L and TEH, C C (2011). Evaluation of beneficial plants as hosts for natural enemies of oil palm bagworms. Proc. of the PIPOC 2011 International Palm Oil Congress (unedited). p. 36-40.
PAVLIK, J (1993). Variability in the host acceptance of European corn borer, Ostrinia nubialis $\mathrm{Hbn}$. (Lepidoptera, Pyralidae) in strains of the egg parasitoid Trichogramma spp. (Hymenoptera, Trichogrammatidae). J. Applied Entomology, 115: 7784.

PEÑA, E R; REYES, R C; BASTIDAS, S P; MORALES, $\mathrm{F}$ and LOZANO, I P (2010). Annular spot and chlorotic ring spot in Elaeis guineensis and OxG hybrids at the nursery stage in Tumaco, Colombia. ASD Oil Palm Papers, 34: 33-45.

SCHIRMER, S; SENGONCA, C and BLAESER, $\mathrm{P}$ (2008). Influence of abiotic factors on some biological and ecological characteristics of the aphid parasitoid Aphelinus asychis (Hymenoptera: Aphelinidae) parasitizing Aphis gossypii (Sternorrhyncha: Aphididae). European J. Entomology, 105(1): 121-129.

SENGONCA, C; BLAESER, P and SCHIRMER, $\mathrm{S}$ (2008). Influence of abiotic factors on some biological and ecological characteristics of the aphid parasitoid Aphelinus asychis (Hymenoptera: Aphelinidae) parasitizing Aphis gossypii (Sternorrhyncha: Aphididae). European J. Entomology, 105: 121-129.

UC IPM, UC Management Guidelines for Lygus Bugs on Eggplant. http://www.ipm.ucdavis.edu/PMG/ r211300611.html

UPADHYAY, R K; MUKERJI, K G and CHAMOLA, B P (2001). Insect pests. Biocontrol Potential and its Exploitation in Sustainable Agriculture. Vol. 2. Springer. $421 \mathrm{pp}$.

WHEELER, A G (2001). Biology of the Plant Bugs (Hemiptera: Miridae): Pests, Predators, Opportunists. Cornell University Press. 507 pp.

ZILAHI-BALOGH, G M G; SHIPP, J L; CLOUTIER, C and BRODEUR, J (2006). Influence of light intensity, photoperiod, and temperature on the efficacy of two aphelinid parasitoids of the Greenhouse Whitefly. Environmental Entomology, 35(3): 581-589. 